\title{
Applications of a finite element discretisation of thin plate splines
}

\author{
Bishnu P. Lamichhane ${ }^{1} \quad$ Linda Stals $^{2}$
}

(Received 1 March 2015; revised 26 November 2015)

\begin{abstract}
The thin plate spline method is a widely used data fitting technique which has the ability to smooth noisy data. We present some example applications of a new mixed finite element discretisation of the thin plate spline method. The new approach works with a pair of bases for the gradient and the Lagrange multiplier forming a biorthogonal system, thus ensuring that the scheme is numerically efficient and the formulation is stable. We overview of the theoretical foundations of the new approach and give numerical examples in both two and three dimensions.
\end{abstract}

http://journal.austms.org.au/ojs/index.php/ANZIAMJ/article/view/9368 gives this article, (c) Austral. Mathematical Soc. 2016. Published January 11, 2016, as part of the Proceedings of the 17th Biennial Computational Techniques and Applications Conference. ISSN 1446-8735. (Print two pages per sheet of paper.) Copies of this article must not be made otherwise available on the internet; instead link directly to this URL for this article. 


\section{Contents}

1 Introduction

C211

2 Discrete setting

C213

3 Algebraic formulation

C215

4 Numerical examples

C216

4.1 Two dimensional examples . . . . . . . . . . . . . . C C217

4.2 Three dimensional examples . . . . . . . . . . . . .

5 Conclusion

$\mathrm{C} 222$

References

$\mathrm{C} 226$

\section{Introduction}

Thin plate splines are used to interpolate and smooth scattered data [4, 12]. Let $\Omega \subset \mathbb{R}^{\mathrm{d}}$ with $\mathrm{d} \in\{2,3\}$ be a polygonal or polyhedral domain. Given a set $\mathcal{G}=\left\{\mathbf{p}_{i}\right\}_{i=0}^{N}$ of scattered points in $\Omega$ and a set of function values $\left\{s_{i}=f\left(p_{i}\right)\right\}_{i=0}^{N}$, the thin plate spline is a smooth function $u: \Omega \rightarrow \mathbb{R}$ which minimises the functional

$$
\frac{1}{N} \sum_{i=1}^{N}\left[u\left(p_{i}\right)-s_{i}\right]^{2}+\alpha \int_{\Omega} \sum_{|v|=2}\left(\begin{array}{l}
2 \\
v
\end{array}\right)\left(D^{v} u\right)^{2} d x
$$

over a space of functions $H^{2}(\Omega)$, where $\boldsymbol{v}=\left(v_{1}, \ldots, v_{d}\right) \in \mathbb{N}_{0}^{d}$ is a $d$ dimensional vector used for multi-index notation, and $\mathrm{D}^{v} u$ denotes the usual partial derivative

$$
\left(\frac{\partial}{\partial x_{1}}\right)^{v_{1}} \cdots\left(\frac{\partial}{\partial x_{d}}\right)^{v_{d}} u
$$


Moreover, $|\boldsymbol{v}|=\sum_{i=1}^{d} v_{i}$ and $\alpha$ is a positive constant. Techniques such as the generalised cross validation (GCV) may be used to find an appropriate choice of $\alpha[6,12]$.

Our formulation is obtained by introducing an auxiliary variable $\boldsymbol{\sigma}=\nabla \mathbf{u}$ such that the minimisation problem (1) is rewritten as $[3,7,9]$

$$
\min _{(\mathfrak{u}, \boldsymbol{\sigma}) \in V, \boldsymbol{\sigma}=\nabla \mathfrak{u}}\left(\frac{1}{\mathrm{~N}} \sum_{i=1}^{\mathrm{N}}\left[u\left(\mathbf{p}_{i}\right)-s_{i}\right]^{2}+\alpha\|\nabla \boldsymbol{\sigma}\|_{\mathrm{L}^{2}(\Omega)}^{2}\right),
$$

where $V=H^{1}(\Omega) \times\left[H^{1}(\Omega)\right]^{\text {d }}$. This is a constrained minimisation problem with the gradient $\boldsymbol{\sigma}$ of the smoother $\boldsymbol{u}$ where both variables are in $\mathrm{H}^{1}$ space. Hence we use a $\mathrm{H}^{1}$-conforming finite element method to discretise this problem, in contrast to the requirement of a $\mathrm{H}^{2}$-conforming method for the minimisation problem (1). A $\mathrm{H}^{1}$-conforming finite element method with a biorthogonal system for the minimisation problem (2) is more efficient than a $\mathrm{H}^{2}$-conforming method for the minimisation problem (1) [11].

We introduce a variational equation for the constraint $\sigma=\nabla u$ by means of a Lagrange multiplier space. Through appropriate choices of bases for the finite element discretisation, the gradient of the smoother $\boldsymbol{\sigma}$ and the Lagrange multiplier $\boldsymbol{\phi}$ are easily eliminated from the discrete system. We introduced this idea previously [10] where we used bubble functions to enrich the discrete space for the gradient to guarantee a unique solution. However, we are unable to prove convergence of this scheme. In our new approach, bubble functions are used to enrich the smoother and we add a consistent stabilising term, similar to the idea described by Arnold and Brezzi [1], to get a stable formulation in the discrete setting.

Lamichhane et al. [11] detailed the theoretical framework for the new approach. The aim of this current article is to give an overview of the techniques and to study example applications in two and three dimensions. 


\section{Discrete setting}

Let $\mathcal{T}_{h}$ be a globally quasi-uniform and shape regular triangulation of the domain $\Omega$ consisting of triangles or tetrahedra with mesh-size h. Let

$$
S_{h}=\left\{u_{h} \in C^{0}(\Omega)\left|u_{h}\right|_{T} \in \mathcal{P}(T), T \in \mathcal{T}_{h}\right\}
$$

be the standard linear finite element space where $\mathcal{P}(\mathrm{T})$ is the space of linear functions on $\mathrm{T}$; and let

$$
B_{h}=\left\{b_{h}\left|b_{h}\right|_{T}=(d+1)^{d+1} \prod_{i=1}^{d+1} \phi_{i}^{\top}, T \in \mathcal{T}_{h}\right\}
$$

be the space of bubble functions where $\left\{\phi_{i}^{\top}\right\}_{i=1}^{d+1}$ is the set of standard linear basis functions associated with the $d+1$ vertices of $T$. Let $L_{h}=S_{h} \oplus B_{h}$. We enrich the standard finite element space $S_{h}$ with element-wise defined bubble functions to obtain the space $L_{h}$. This is done in order to obtain an optimal error estimate of the discrete solution.

Denoting the discrete counterpart of the continuous space $V$ by $V_{h}=L_{h} \times\left[S_{h}\right]^{d}$, a discrete version of $(2)$ is

$$
\min _{\left(u_{h}, \boldsymbol{\sigma}_{h}\right) \in V_{h}}\left(\frac{1}{N} \sum_{i=0}^{N}\left[u_{h}\left(p_{i}\right)-s_{i}\right]^{2}+\alpha\left\|\nabla \sigma_{h}\right\|_{L^{2}(\Omega)}^{2}\right)
$$

subject to the constraint

$$
\left\langle\boldsymbol{\sigma}_{\mathrm{h}}, \boldsymbol{\psi}_{\mathrm{h}}\right\rangle_{\mathrm{L}^{2}(\Omega)}=\left\langle\nabla \boldsymbol{u}_{\mathrm{h}}, \boldsymbol{\psi}_{\mathrm{h}}\right\rangle_{\mathrm{L}^{2}(\Omega)}, \quad \boldsymbol{\psi}_{\mathrm{h}} \in\left[\mathrm{M}_{\mathrm{h}}\right]^{\mathrm{d}} .
$$

The space $\left[M_{h}\right]^{d}$ plays the role of a Lagrange multiplier space. As discussed in Section 3, $M_{h} \subset L^{2}(\Omega)$ is constructed in such a way as to simplify the resulting saddle point problem.

We previously used a similar approach [10] but found the saddle point problem is not stable since the discretisation is similar to a $P_{1}-P_{1}$ discretisation of 
a Darcy or Stokes problem [5, 8, for example]. To avoid this problem we now introduce a modification, as suggested by Arnold and Brezzi [1] for the Mindlin-Reissner plate, so that our discrete saddle point problem is to find $\left[\left(u_{h}, \sigma_{h}\right), \Phi_{h}\right] \in V_{h} \times\left[M_{h}\right]^{d}$ such that

$$
\begin{aligned}
\mathrm{A}\left[\left(\mathrm{u}_{\mathrm{h}}, \boldsymbol{\sigma}_{\mathrm{h}}\right),\left(v_{\mathrm{h}}, \boldsymbol{\tau}_{\mathrm{h}}\right)\right]+\mathrm{B}\left[\boldsymbol{\phi}_{\mathrm{h}},\left(v_{\mathrm{h}}, \boldsymbol{\tau}_{\mathrm{h}}\right)\right] & =\mathrm{f}\left(v_{\mathrm{h}}\right), \quad\left(v_{\mathrm{h}}, \boldsymbol{\tau}_{\mathrm{h}}\right) \in \mathrm{V}_{\mathrm{h}}, \\
\mathrm{B}\left[\boldsymbol{\psi}_{\mathrm{h}},\left(\mathrm{u}_{\mathrm{h}}, \boldsymbol{\sigma}_{\mathrm{h}}\right)\right] & =0, \quad \boldsymbol{\psi}_{\mathrm{h}} \in\left[\mathrm{M}_{\mathrm{h}}\right]^{\mathrm{d}},
\end{aligned}
$$

where

$$
\begin{aligned}
A\left[\left(u_{h}, \boldsymbol{\sigma}_{h}\right),\left(v_{h}, \boldsymbol{\tau}_{h}\right)\right]= & \frac{1}{\mathrm{~N}}\left(\mathrm{P} u_{\mathrm{h}}\right)^{\mathrm{T}} \mathrm{P} v_{\mathrm{h}}+\alpha \int_{\Omega} \nabla \boldsymbol{\sigma}_{\mathrm{h}}: \nabla \boldsymbol{\tau}_{\mathrm{h}} \mathrm{d} \boldsymbol{x}, \\
& +\mathrm{r} \int_{\Omega}\left(\boldsymbol{\sigma}_{\mathrm{h}}-\nabla \mathrm{u}_{\mathrm{h}}\right) \cdot\left(\boldsymbol{\tau}_{\mathrm{h}}-\nabla v_{\mathrm{h}}\right) \mathrm{d} \boldsymbol{x}, \\
\mathrm{B}\left[\boldsymbol{\psi}_{\mathrm{h}},\left(v_{\mathrm{h}}, \boldsymbol{\tau}_{\mathrm{h}}\right)\right]= & \int_{\Omega} \boldsymbol{\tau}_{\mathrm{h}} \cdot \boldsymbol{\psi}_{\mathrm{h}} \mathrm{d} \boldsymbol{x}-\int_{\Omega} \nabla v_{\mathrm{h}} \cdot \boldsymbol{\psi}_{\mathrm{h}} \mathrm{d} \boldsymbol{x}, \\
\mathrm{f}\left(v_{\mathrm{h}}\right)= & \frac{1}{\mathrm{~N}}\left(\mathrm{P} \boldsymbol{v}_{\mathrm{h}}\right)^{\mathrm{T}} \mathbf{s},
\end{aligned}
$$

with $r>0$ a stabilisation parameter. Here we denote function values of $u$ at the measurement points $\left\{\mathbf{p}_{i}\right\}_{i=0}^{N}$ by $P \mathfrak{u}=\left(\mathfrak{u}\left(\mathbf{p}_{0}\right), \mathfrak{u}\left(\mathbf{p}_{1}\right), \ldots, \mathfrak{u}\left(\mathbf{p}_{\mathrm{N}}\right)\right)^{\top}$ and $\mathbf{s}$ is a column vector with ith entry $s_{i}$ for $i=0, \ldots, N$. The notation $\nabla \boldsymbol{\sigma}_{\mathrm{h}}: \nabla \boldsymbol{\tau}_{\mathrm{h}}$ denotes the dot product of the two matrices $\nabla \sigma_{h}$ and $\nabla \tau_{h}$. The integral multiplied by $r$ is a stabilisation term. The remaining terms in $A[\cdot, \cdot]$ come from $(4)$ and $\mathrm{B}[\cdot, \cdot \cdot]$ is a bilinear form of constraint (5).

Since the stabilisation term is consistent, the parameter $r>0$ is in principle arbitrary. By choosing an appropriate value of $r$, the stabilisation can accelerate the solver, as in an augmented Lagrangian formulation [2].

The error estimate is obtained in the energy norm $\|\cdot\|_{A}$ induced by the bilinear form $A[\cdot, \cdot]$ and is defined as

$$
\|(\mathfrak{u}, \boldsymbol{\sigma})\|_{\mathcal{A}}:=\sqrt{\frac{1}{N}\|\mathrm{Pu}\|^{2}+\alpha|\boldsymbol{\sigma}|_{\mathrm{H}^{1}(\Omega)}^{2}+r\|\boldsymbol{\sigma}-\nabla u\|_{\mathrm{L}^{2}(\Omega)}^{2}},
$$


where $(\boldsymbol{u}, \boldsymbol{\sigma}) \in\left(\mathrm{C}^{0}(\Omega) \cap \mathrm{H}^{1}(\Omega)\right) \times\left[\mathrm{H}^{1}(\Omega)\right]^{\mathrm{d}}$. Lamichhane et al. [11] showed that the difference between the solution of the original continuous problem (1) and the solution of the discrete problem (6) decreases linearly with the mesh-size $h$ in the energy norm $\|\cdot\|_{A}$. See Theorem 4.4, Theorem 4.6 and Collorary 4.7 by Lamachhane et al. [11] for a description of the framework needed to prove such a convergence result.

\section{$3 \quad$ Algebraic formulation}

The following lists matrices and their associated bilinear form:

$$
\begin{aligned}
& \mathrm{R}: \quad \frac{1}{\mathrm{~N}}\left(\mathrm{P} \mathfrak{u}_{\mathrm{h}}\right)^{\mathrm{T}} \mathrm{P} \boldsymbol{v}_{\mathrm{h}}, \quad \mathrm{A}: \int_{\Omega} \nabla \boldsymbol{\sigma}_{\mathrm{h}}: \nabla \boldsymbol{\tau}_{\mathrm{h}} \mathrm{d} \boldsymbol{x}, \\
& \text { B : } \int_{\Omega} \nabla u_{h} \cdot \boldsymbol{\psi}_{\mathrm{h}} \mathrm{d} \boldsymbol{x}, \quad W: \int_{\Omega} \nabla \mathfrak{u}_{\mathrm{h}} \cdot \boldsymbol{\tau}_{\mathrm{h}} \mathrm{d} \boldsymbol{x} \text {, } \\
& \mathrm{K}: \int_{\Omega} \nabla \mathrm{u}_{\mathrm{h}} \cdot \nabla v_{\mathrm{h}} \mathrm{d} \boldsymbol{x}, \quad \mathrm{D}: \int_{\Omega} \sigma_{\mathrm{h}} \cdot \boldsymbol{\psi}_{\mathrm{h}} \mathrm{d} \boldsymbol{x} \text {, } \\
& M: \int_{\Omega} \sigma_{\mathrm{h}} \cdot \tau_{\mathrm{h}} \mathrm{d} x \text {. }
\end{aligned}
$$

We use the vectors $\mathrm{U}, \boldsymbol{\Sigma}$ and $\boldsymbol{\Phi}$ to store the coefficients associated with the discrete variables $\boldsymbol{u}_{h}, \boldsymbol{\sigma}_{h}$ and $\boldsymbol{\phi}_{h}$, respectively.

The first equation of (6) is true for all test functions $\boldsymbol{\tau}_{\mathrm{h}}$ and $\boldsymbol{\nu}_{\mathrm{h}}$, so by setting $\tau_{\mathrm{h}}$ and $\nu_{\mathrm{h}}$ to zero,

$$
\begin{gathered}
\frac{1}{N}\left(\mathrm{P} u_{h}\right)^{T} P v_{h}-\int_{\Omega} \nabla v_{h} \cdot \phi_{h} \mathrm{~d} x-r \int_{\Omega}\left(\sigma_{h}-\nabla u_{h}\right) \cdot \nabla v_{h} \mathrm{~d} x=f\left(v_{h}\right), \\
\alpha \int_{\Omega} \nabla \sigma_{h}: \nabla \tau_{h} \mathrm{~d} x+\int_{\Omega} \boldsymbol{\phi}_{h} \cdot \boldsymbol{\tau}_{h} \mathrm{~d} x+r \int_{\Omega}\left(\sigma_{h}-\nabla u_{h}\right) \cdot \tau_{h} \mathrm{~d} x=0,
\end{gathered}
$$

with $v_{\mathrm{h}} \in \mathrm{L}_{\mathrm{h}}$ and $\boldsymbol{\tau}_{\mathrm{h}} \in\left[\mathrm{S}_{\mathrm{h}}\right]^{\mathrm{d}}$. Then the saddle point problem (6) is written as the linear system

$$
\left[\begin{array}{ccc}
\mathrm{R}+\mathrm{rK} & -\mathrm{rW} W^{\top} & -\mathrm{B}^{\top} \\
-\mathrm{rW} & \alpha \mathrm{A}+\mathrm{rM} & \mathrm{D}^{\top} \\
-\mathrm{B} & \mathrm{D} & 0
\end{array}\right]\left[\begin{array}{c}
\mathrm{U} \\
\Sigma \\
\Phi
\end{array}\right]=\left[\begin{array}{l}
\mathrm{F} \\
0 \\
0
\end{array}\right]
$$


where $F$ is the discrete vector form of the linear form $f(\cdot)$.

Recall $S_{h}$ is the standard finite element basis space, $M_{h}$ is defined such that $\operatorname{dim} M_{h}=\operatorname{dim} S_{h}$, and the basis of $S_{h}$ and $M_{h}$ satisfy a biorthogonality relationship. Lamichhane et al. [11] defined exactly the definition of the basis of $M_{h}$.

Constructing $M_{h}$ so that a biorthogonality condition is satisfied ensures that $\mathrm{D}$ is a nonsingular diagonal matrix. This allows us to to statically condense out the degree of freedom associated with $\boldsymbol{\Sigma}$ and $\boldsymbol{\Phi}$. After statically condensing out variables $\boldsymbol{\Sigma}$ and $\boldsymbol{\Phi}$ (block elimination) from (8), we arrive at the reduced system

$$
\left[(R+r K)-r\left(W^{\top} D^{-1} B+B^{\top} D^{-1} W\right)+B^{\top} D^{-1}(\alpha A+r M) D^{-1} B\right] U=F .
$$

Lamichhane et al. [11, Theorem 4.1] proved that the system in (9) is positive definite if the domain contains at least three non-collinear points for $\mathrm{d}=2$ and four non-coplanar points for $\mathrm{d}=3$.

\section{Numerical examples}

To finish the discussion we now present some example applications of the method described above. In all of the examples the domain is the unit square and the finite element grid is a uniform grid. This approach was taken for ease of implementation; the theory does not require the use of a uniform finite element grid.

We used the preconditioned conjugate gradient method to solve equation (9). The preconditioner is described by Lamichhane et al. [11]. The iterations continue until the $l_{2}$ norm of the residual is less than $10^{-7}$. To find an initial guess we fit the linear polynomial $p_{1}(\boldsymbol{x})=\mathrm{c}_{0}+\sum_{i=1}^{d} c_{i} x_{i}$ to the original data set by using the standard least squares method. The reason for using the 
linear polynomial as an initial guess is to avoid issues that may arise when $\alpha$ is large or there are a small number of data points.

There is a trade-off in choosing an appropriate value for $r$. If $r$ is too large for a given value of $h$, then the spline will be too smooth. On the other hand, as discussed in Section 2, the stabilisation term is required to obtain a consistent system, so $r$ cannot be made arbitrarily small. We set $r=10^{4} \alpha$.

\subsection{Two dimensional examples}

The first example we look at is the Matlab spine data set (which is obtained by typing load spine in a Matlab command window). The data set consists of 179830 points. The top plot of Figure 1 contains an image plot of the original data set. The two images in Figure 2 show an image plot of $\mathfrak{u}_{\mathfrak{h}}\left(\mathbf{p}_{i}\right)$ with $0 \leqslant i \leqslant N$ for different sized finite element grids. The bottom plot of Figure 1 shows a plot of $\left|\left(\mathfrak{u}_{\mathfrak{h}}\left(\boldsymbol{p}_{\mathfrak{i}}\right)-s_{\mathfrak{i}}\right)\right|$ for $0 \leqslant i \leqslant N$. Not surprisingly, most of the smoothing occurred around the edges of the bone structure.

The top plot of Figure 3 presents a surface plot of the original data set and the bottom plot is the finite element approximation. These give a different perspective when comparing the original data set and the spline approximation.

The next test problem is artificially generated. It is designed to test what happens when data is missing. We initially took $99 \times 99$ data points evenly distributed on the domain $0.01 \leqslant\left\|p_{i}\right\|_{\infty} \leqslant 0.99$ and assigned each point the value $s_{i}=\sin \left(4 \pi x_{i}\right) \sin \left(4 \pi y_{i}\right)$ where $p_{i}=\left(x_{i}, y_{i}\right)$. The top plot of Figure 4 shows this data set. The bottom plot of Figure 4 shows the surface plot of the finite element approximation to the spline on a grid with 4225 vertices and $\alpha=10^{-7}$. We see that regions of missing data are filled with a smooth spline.

To create the last test problem considered in this section we took the data set in the top plot of Figure 4 and added 5\% uniformally distributed noise, 
Figure 1: Image plots of (top) the Matlab spine data set and (bottom) the difference between the original spine data set and the finite element approximation of the spine data set using a finite element grid with 66049 vertices and $\alpha=10^{-9}$.
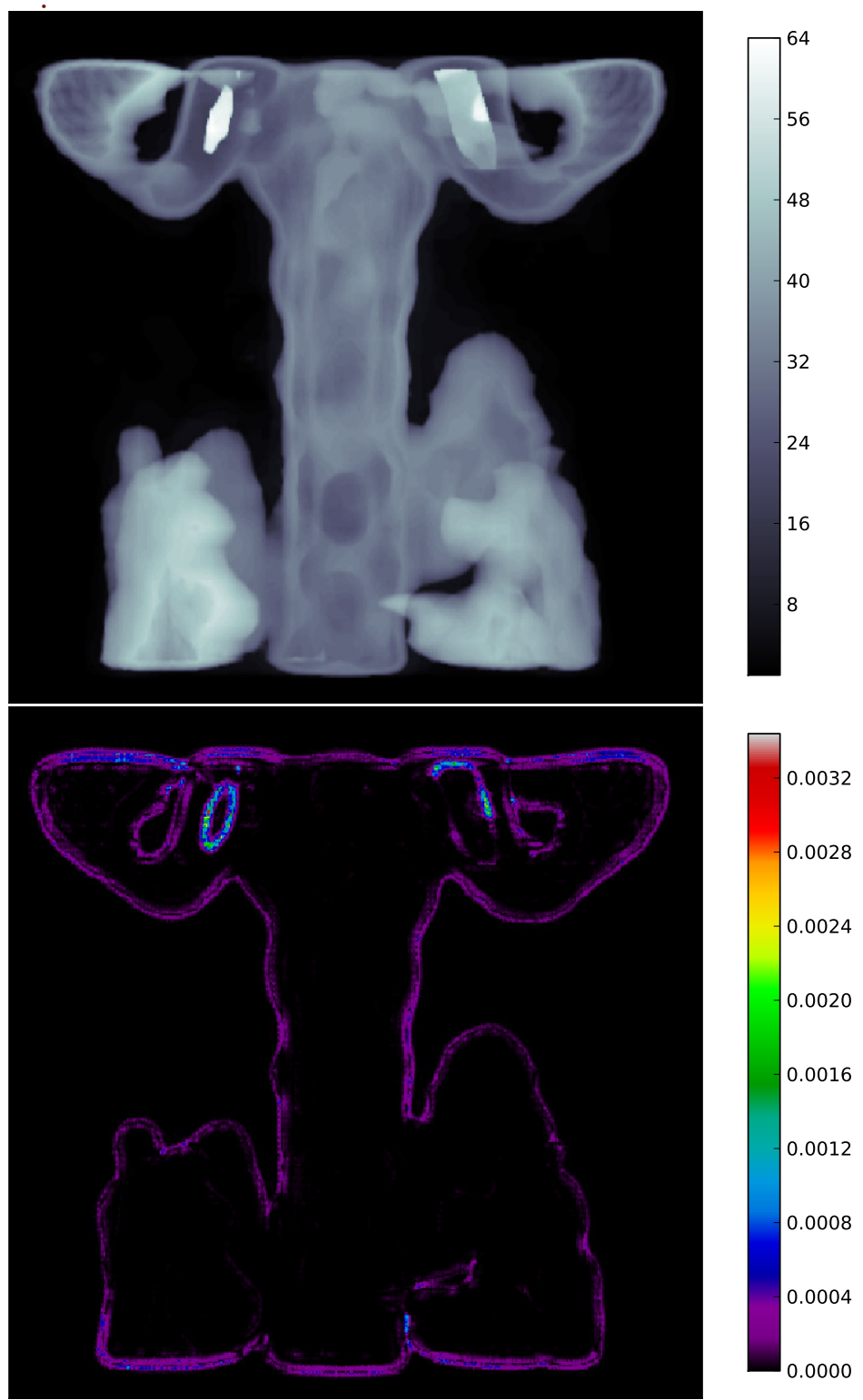
Figure 2: Image plots of the finite element approximation of the spine data set with $\alpha=10^{-9}$ and using a finite element grid with (top) 289 vertices and (bottom) 4225 vertices
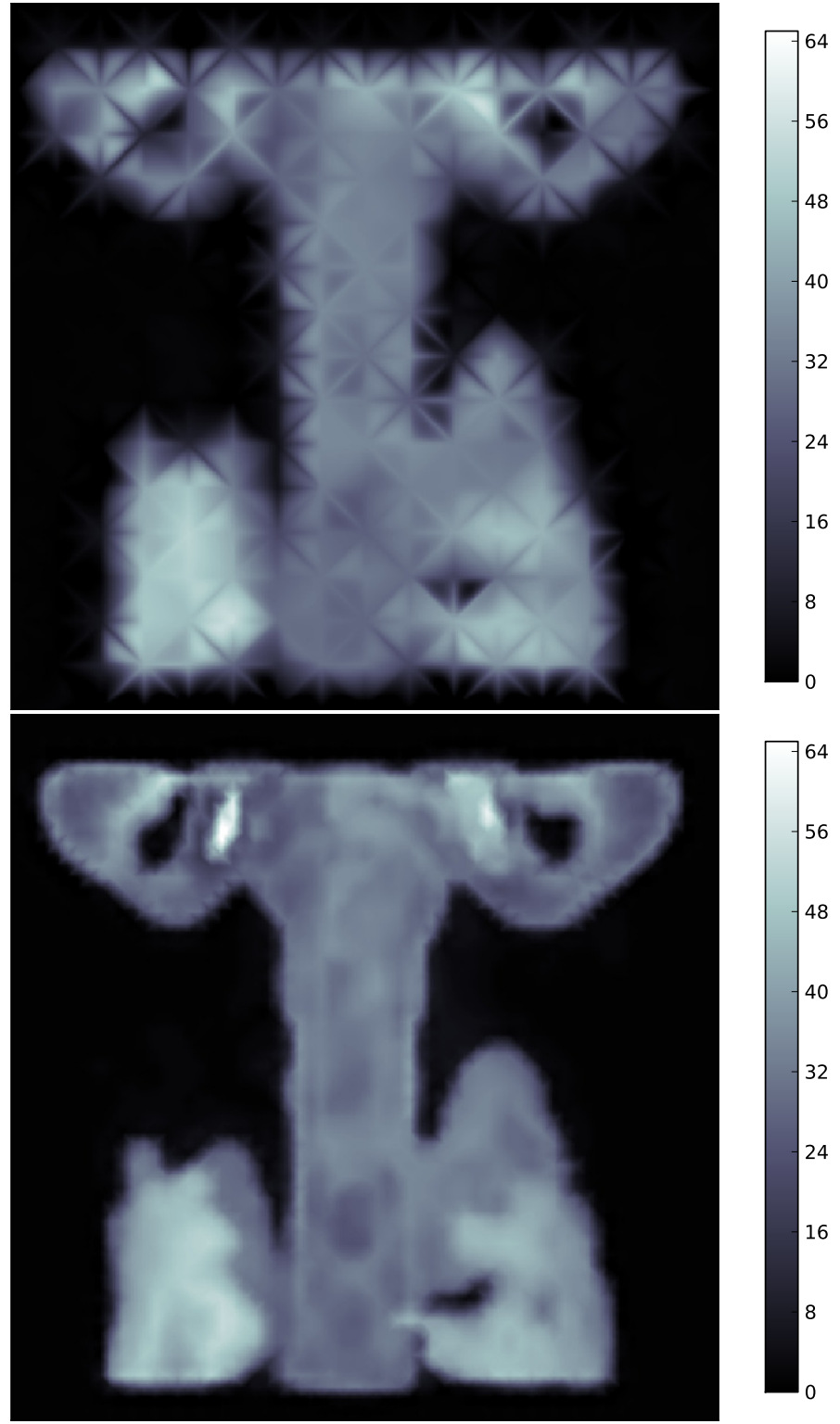
Figure 3: Surface plots of (top) the Matlab spine data set and (bottom) its finite element approximation using a grid with 66049 vertices and $\alpha=10^{-9}$.
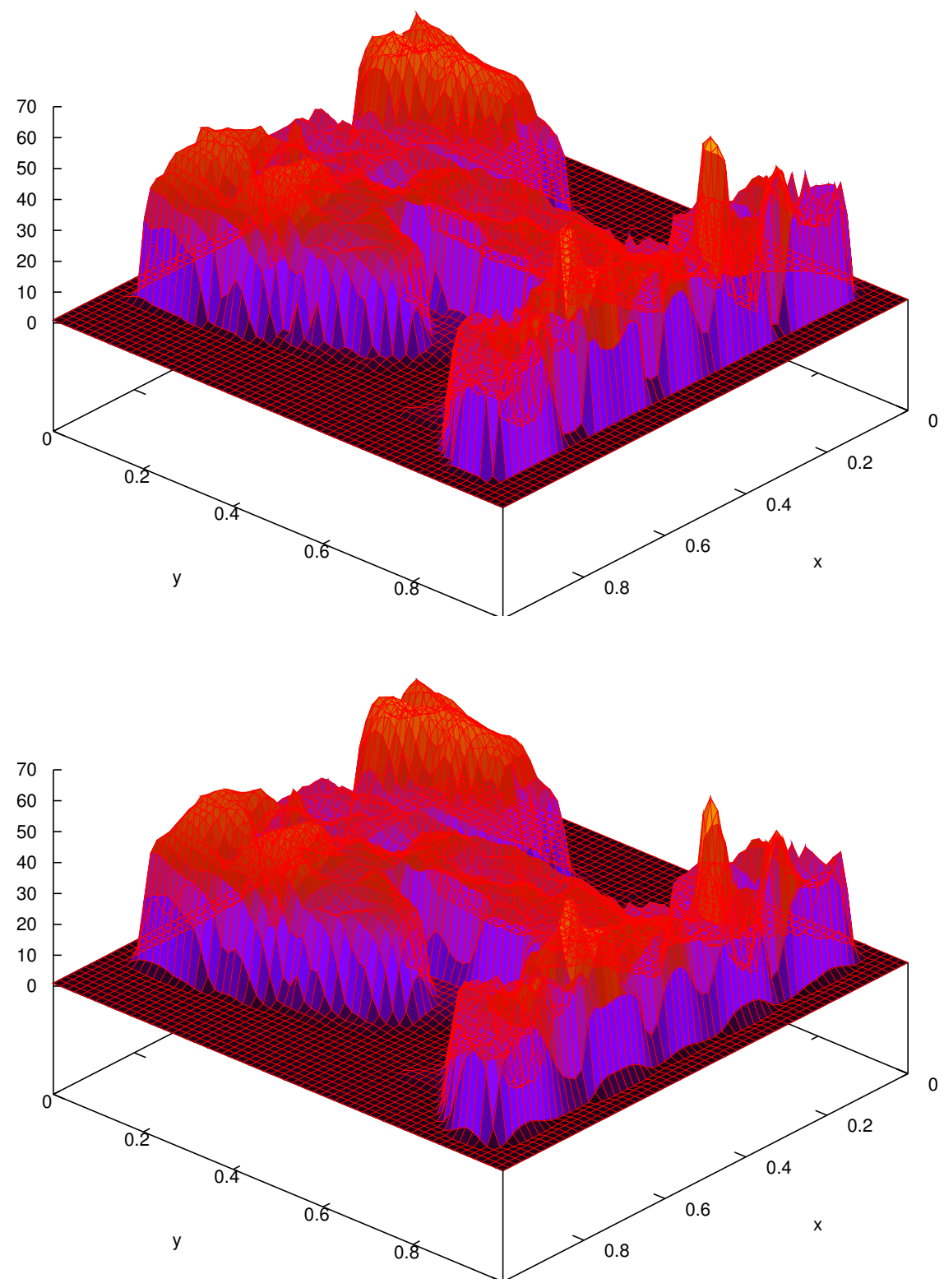
Figure 4: Surface plots of (top) the sine data set and (bottom) its finite element approximation using a finite element grid with 4225 vertices and $\alpha=10^{-7}$.
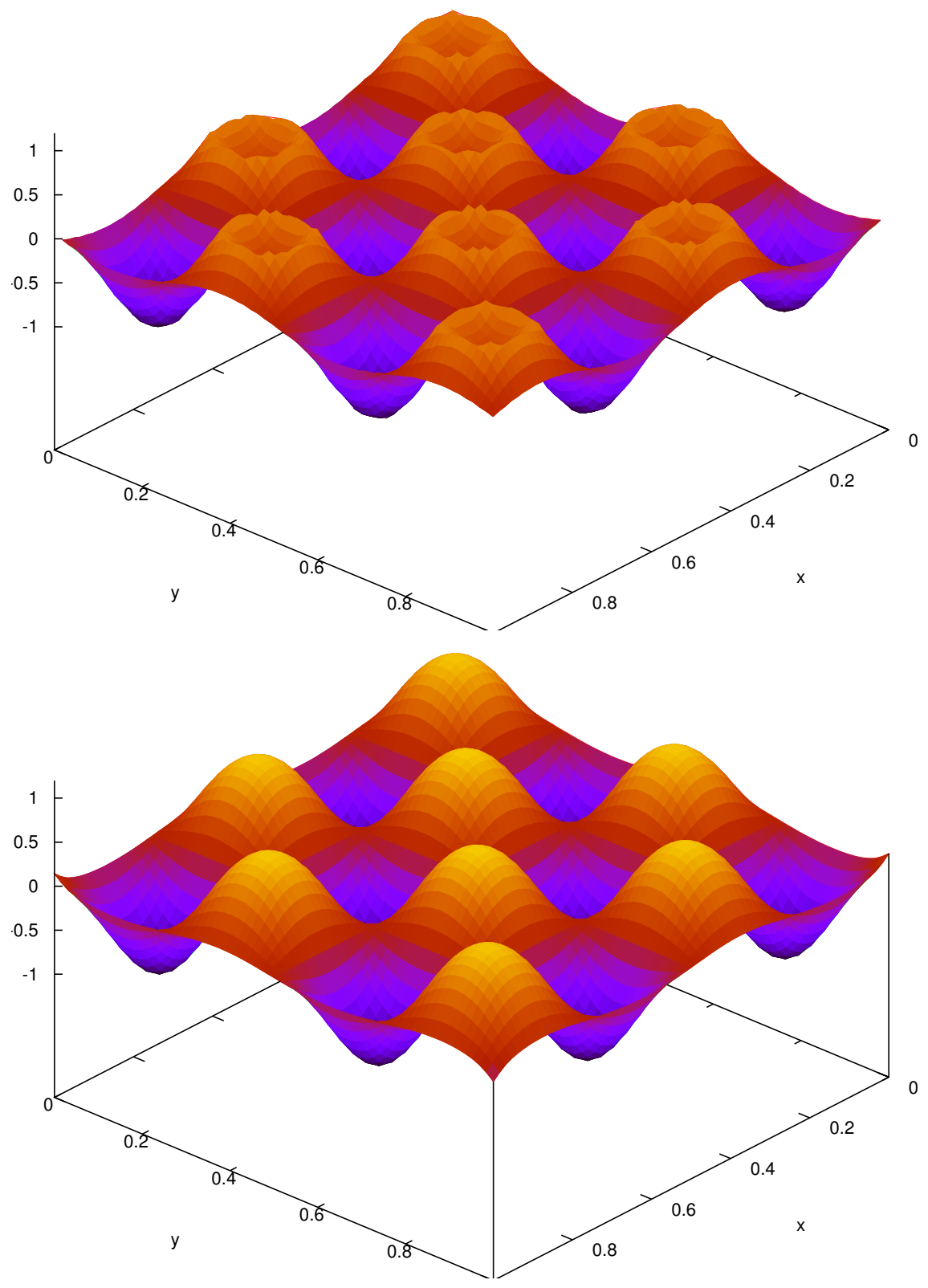
producing the top plot of Figure 5. The bottom plot of Figure 5 shows the surface plot of the finite element approximation to the spline on a grid using 4225 vertices with $\alpha=10^{-8}$. The noise is evident around the boundary of the domain of the bottom plot, but appears to be smoothed out in the interior.

\subsection{Three dimensional examples}

For the three dimensional example we took $101 \times 101 \times 101$ data points evenly distributed on the domain $10 / 101 \leqslant\left\|p_{i}\right\|_{\infty} \leqslant 10-10 / 101$ and assigned each point the value $s_{i}=\sin \left(x_{i} y_{i} z_{i}\right) /\left(x_{i} y_{i} z_{i}\right)$ where $p_{i}=\left(x_{i}, y_{i}, z_{i}\right)$. The top right plot of Figure 6 shows the data set. Isosurface plots of the finite element approximation to the spline on grids with different numbers of nodes are in the two bottom plots of Figure 6 .

The data set used in Figure 6 describes a smooth function. Now we look at an example of a noisy data set. We take the data set in the top right plot of Figure 6 and add 5\% uniformly distributed random noise. An isosurface plot of the noisy data set is shown in the top right plot of Figure 7. The plotting routine applies some smoothing to the data set, so the figure does not show all the detail. The thin plate spline uses the biharmonic equation to smooth out the noise in the data set, as shown in the two bottom plots of Figure 7.

\section{Conclusion}

A mixed finite element method for approximating thin plate splines in two and three dimensions was presented. The particular choice of finite element bases was determined by the aim to simplify the resulting system of equations. A stabilising term was introduced into the system to ensure that it is stable. A number of example applications were given to explore the properties of this new approach. 
Figure 5: Surface plots of (top) noisy sine data and (bottom) its finite element approximation using a finite element grid with 4225 vertices and $\alpha=10^{-8}$.
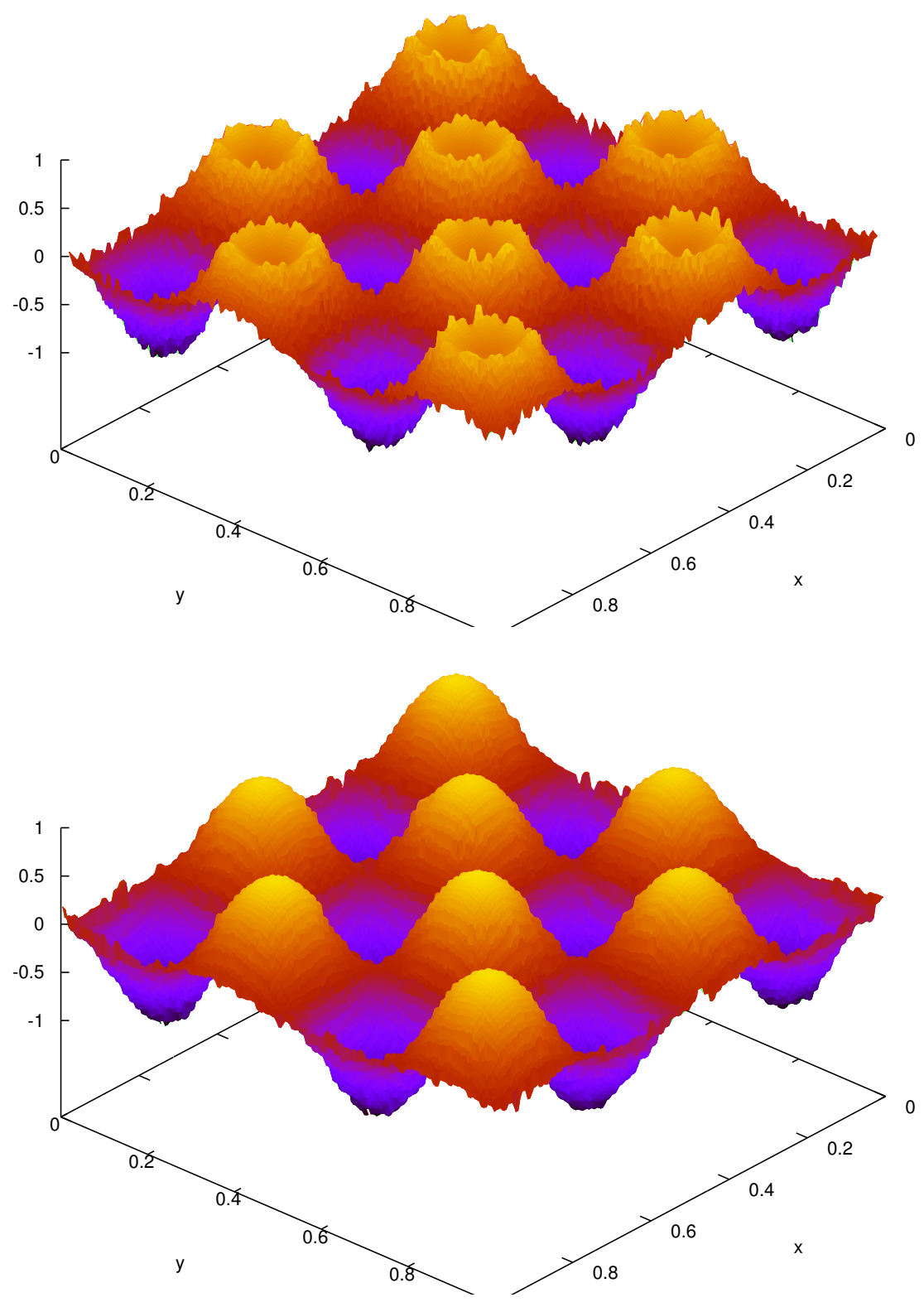
Figure 6: Isosurface plots of the 3D test example (top right) without noise, and its finite element approximations with $\alpha=10^{-7}$ and using a finite element grid and (bottom left) 2465 vertices and (bottom right) 17985 vertices.
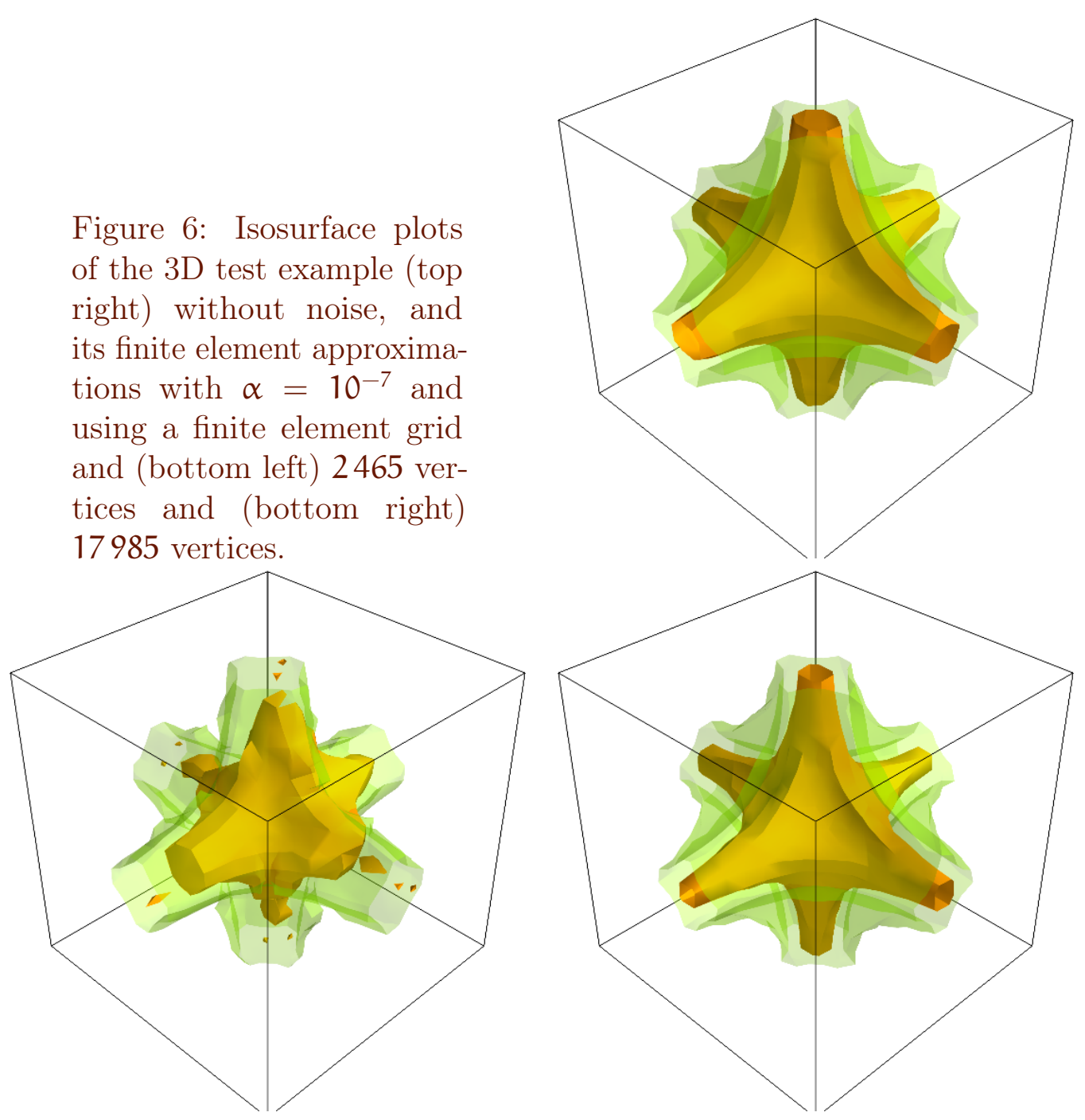
Figure 7: Isosurface plots of the 3D test example (top right) with noise, and its finite element approximations with $\alpha=10^{-7}$ and using a finite element grid with (bottom left) 2465 vertices and (bottom right) 17985 vertices.
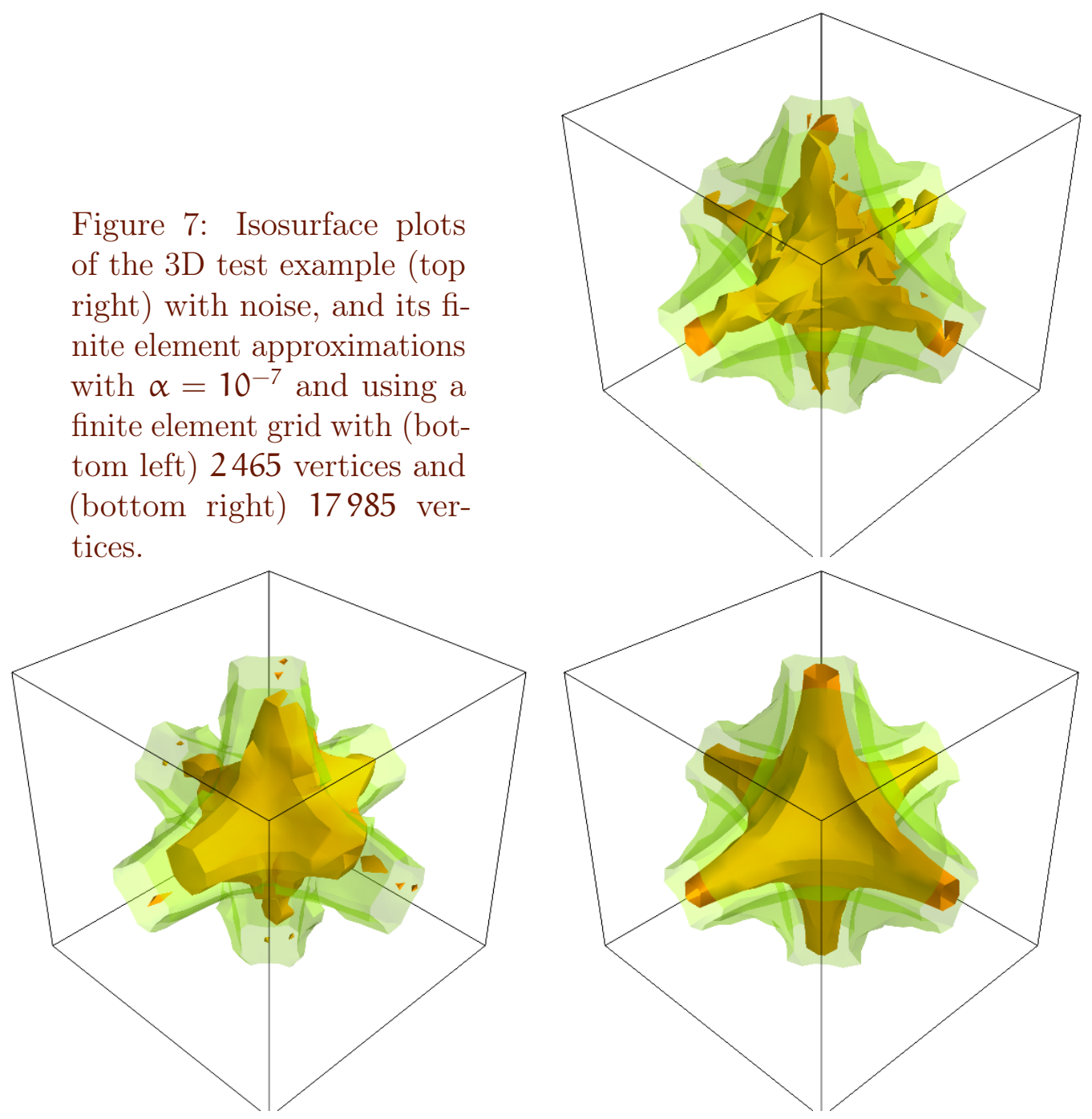


\section{References}

[1] D. N. Arnold and F. Brezzi. Some new elements for the Reissner-Mindlin plate model. In Boundary Value Problems for Partial Differential Equations and Applications, pages 287-292. Masson, Paris, 1993. C212, C214

[2] D. Boffi and C. Lovadina. Analysis of new augmented Lagrangian formulations for mixed finite element schemes. Numer. Math., 75:405-419, 1997. doi:10.1007/s002110050246 C214

[3] X.-L. Cheng, W. Han, and H.-C. Huang. Some mixed finite element methods for biharmonic equation. J. Comput. Appl. Math., 126:91-109, 2000. doi:10.1016/S0377-0427(99)00342-8 C212

[4] J. Duchon. Splines minimizing rotation-invariant semi-norms in Sobolev spaces. In Constructive Theory of Functions of Several Variables, Lecture Notes in Mathematics, 571:85-100. Springer-Verlag, Berlin, 1977. doi:10.1007/BFb0086566 C211

[5] V. Girault and P.-A. Raviart. Finite Element Methods for Navier-Stokes Equations. Springer-Verlag, Berlin, 1986. doi:10.1007/978-3-642-61623-5 C214

[6] M. F. Hutchinson. A stochastic estimator of the trace of the influence matrix for Laplacian smoothing splines. Commun. Stat. Simulat. Comput., 19:433-450, 1990. doi:10.1080/03610919008812866 C212

[7] C. Johnson and J. Pitkäranta. Analysis of some mixed finite element methods related to reduced integration. Math. Comput., 38(158):375-400, 1982. doi:10.2307/2007276 C212

[8] T. Karper, K.-A. Mardal, and R. Winther. Unified finite element discretizations of coupled Darcy-Stokes flow. Numer. Meth. Part. D. E., 25:311-326, 2009. doi:10.1002/num.20349 C214 
[9] B. P. Lamichhane. A stabilized mixed finite element method for the biharmonic equation based on biorthogonal systems. J. Comput. Appl. Math., 235:5188-5197, 2011. doi:10.1016/j.cam.2011.05.005 C212

[10] B. P. Lamichhane, S. G. Roberts, and L. Stals. A mixed finite element discretisation of thin-plate splines. In W. McLean and A. J. Roberts (Eds), Proceedings of the 15th Biennial Computational Techniques and Applications Conference, CTAC-2010, ANZIAM J., 52:C518-C534, 2010. http://journal.austms.org.au/ojs/index.php/ANZIAMJ/article/ view/3934 C212, C213

[11] B. P. Lamichhane, S. G. Roberts, and L. Stals. A mixed finite element discretisation of thin plate splines based on biorthogonal systems. J. Sci. Comput., 1-23, July 2015. doi:10.1007/s10915-015-0068-6 C212, C215, $\mathrm{C} 216$

[12] G. Wahba. Spline Models for Observational Data, volume 59 of Series in Applied Mathematic. SIAM, Philadelphia, 1990. doi:10.1137/1.9781611970128 C211, C212

\section{Author addresses}

1. Bishnu P. Lamichhane, School of Mathematical and Physical Sciences, The University of Newcastle, Callaghan NSW 2308, Australia mailto:Bishnu.Lamichhane@newcastle.edu.au

2. Linda Stals, Department of Mathematics, Australian National University, Canberra ACT 0200, Australia mailto:Linda.Stals@anu. edu. au 